\title{
Lupus erythematosus panniculitis
}

\author{
Alizade Narges ${ }^{1}$, Rafiei Rana ${ }^{1}$, Mesbah Alireza$^{2}$, Naji Rad Sara $^{3}$ \\ ${ }^{1}$ Department of Dermatology, Skin Research Center, Guilan University of Medical Sciences, Razi Hospital, Rasht, Iran, \\ ${ }^{2}$ Department of Pathology, Skin Research Center, Guilan University of Medical Sciences, Razi Hospital, Sardare Jangal \\ Street, Rasht, Iran, ${ }^{3}$ Department of Internal Medicine, Internal Medicine Resident, Nassau University Medical Center, \\ New York, USA
}

Corresponding author: Dr. Rafiei Rana, E-mail: rafieirana2@gmail.com

\begin{abstract}
Lupus erythematosus panniculitis is a rare type of chronic cutaneous lupus erythematosus which is histopathologically characterized by a lobular panniculitis. We present a 43-year-old woman with an indurated painful plaque on her right flank from 3 years ago. She mentioned a previous blunt trauma to this site. Microscopic examination revealed a predominant lobular panniculitis with lymphoid follicle formation, hyaline fat necrosis, membranocystic changes and mucin deposition. She was treated with oral hydroxychloroquine with moderate improvement. An indurated subcutaneous painful plaque could be the presenting feature of lupus panniculitis and we should evaluate systemic involvement in these patients.
\end{abstract}

Key words: Lupus panniculitis; Lobular panniculitis; Lymphoid follicle

\section{INTRODUCTION}

Lupus erythematosus panniculitis (LEP) is a subtype of cutaneous lupus erythematosus which is also named lupus erythematosus profundus. Histopathologically, LEP could be characterized by epidermal changes similar to discoid lupus erythematosus (DLE) and a periseptal or lobular panniculitis [1,2].

Lymphoid follicle formation, dermal mucin deposition, hyaline fat necrosis and fibrosclerosis are other characteristic features which have been reported [2,3]. LEP usually presents by subcutaneous nodules, indurated plaques or ulcerations which involves face, proximal extremities and breast $[1,3,4]$.

\section{CASE PRESENTATION}

We report a 43-year-old woman who developed an indurated painful pigmented plaque on her right flank. It was started 3 years ago after a blunt trauma with no improvement and gradually enlarged (Fig. 1).
In past medical history, she had gastritis, hypothyroidism and polycystic ovary syndrome. Drug history included levothyroxine, lansoprazole and contraceptive pills.

In physical examination there was a pigmented deeply seated plaque with woody consistency on the right flank, measured $10 \mathrm{~cm} \times 5 \mathrm{~cm}$. Also there was periungual erythema without cuticular telangiectasia on her hands. There was no oral ulcer, cicatricial alopecia, lymphadenopathy, organomegaly, malar rash, muscle weakness, fever, weight loss and arthritis but she mentioned photosensitivity, morning stiffness, arthralgia and Raynaud's phenomenon.

Skin biopsy was made with patient's consent. Differential diagnoses were deep morphea, lupus panniculitis, sarcoidosis, subcutaneous T cell lymphoma (SCTCL), tuberculosis and cutaneous metastasis.

Histopathologic changes in hematoxylin and eosin (H\&E) staining were as follows: Unremarkable epidermis, pigment incontinence, perivascular, periadnexal and perineural lymphocytic infiltration 
in dermis, lymphoid follicles with germinal centers associated with plasmacytic and neutrophilic infiltration in deep dermis and subcutaneous fat, lobular panniculitis with hyaline fat necrosis, septal thickening and membranocystic lipodystrophy. (Figs. 2a-2e).

Periodic Acid Schiff (PAS) staining revealed mild and focal thickening of subepidermal basement membrane. Mucin deposition was detected in colloidal iron staining. There was no acid fast bacillus in Ziehl Neelsen staining. In immunofluorescence studies

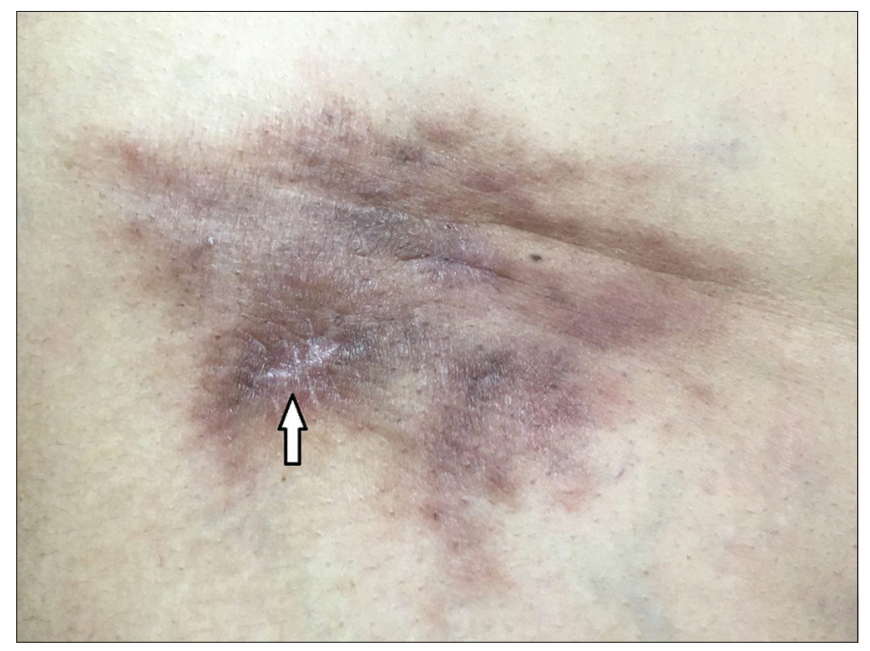

Figure 1: An indurated hyperpigmented plaque on the right flank. Arrowhead points skin biopsy site. only trace and focal deposits of IgG along the dermoepidermal junction were reported (Figs. $2 \mathrm{f}-2 \mathrm{~h}$ ).

Immunohistochemistry (IHC) studies with CD3 (pan T cell marker), CD20 (pan B cell marker), Ki67, CD68 staining were made to differentiate between LEP and subcutaneous T-cell lymphoma. The IHC confirmed mixed lymphoid aggregations of CD20 positive B lymphocytes associated with $\mathrm{CD} 3$ positive T lymphocytes. Ki67 staining revealed germinal centers with expected elevated proliferation rate. CD68 was positive in scattered background histiocytes and follicular dendritic cells but there was no granuloma formation. According to these findings, SCTCL was ruled out (Figs. $3 \mathrm{a}-3 \mathrm{~d})$.

Blood investigations including complete blood count, urea, creatinine, liver function test, calcium, phosphorus, creatine phosphokinase, lactate dehydrogenase and complement levels were within normal limits but following lab data were elevated: Erythrocyte sedimentation rate $(54 \mathrm{~mm} / \mathrm{l} \mathrm{hr}$.), $\mathrm{C}$ - reactive protein ( $15 \mathrm{mg} /$; norm up to 6), antinuclear antibody (ANA: 1/160 titer with homogenous pattern), anti-double stranded DNA antibody $(45 \mathrm{U} / \mathrm{ml}$, norm up to 25$)$ and angiotensin converting enzyme $(83 \mathrm{U} / \mathrm{L}$, norm up to 67). Urine analysis revealed leukocyturia and trace proteinuria $(15 \mathrm{mg} / \mathrm{dl})$. Urine protein was

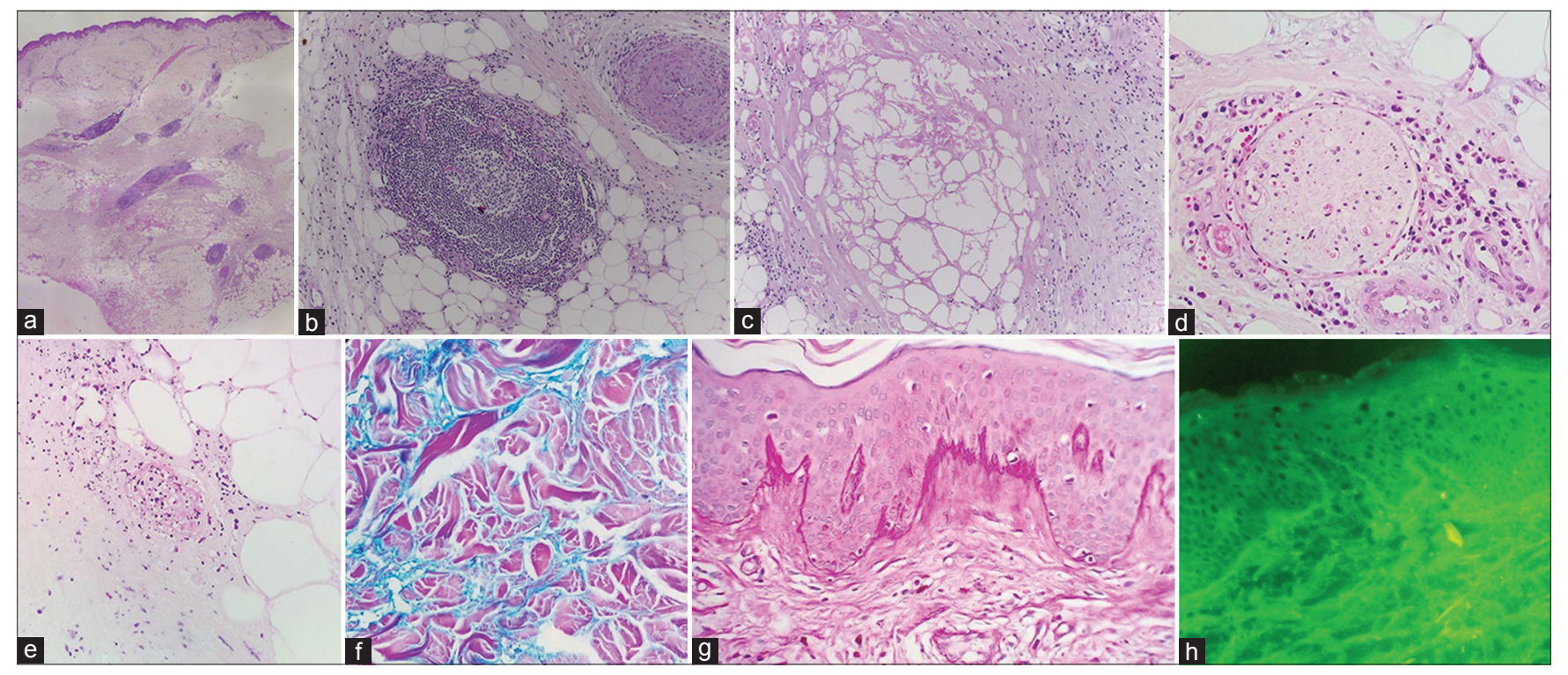

Figure 2: These photomicrographs show: a: Perivascular and periadnexal lymphocytic infiltration in dermis, dense lymphoid follicle aggregations in deep dermis and subcutaneous fat, lobular panniculitis and septal thickening in sub cutaneous fat. b: lymphoid follicle with germinal center associated with plasmacytic infiltration in subcutaneous fat. c: membranocystic lipodystrophy with arabesque structures and hyaline fat necrosis. $\mathrm{d}$ : Lymphocytic and plasmacytic perineural infiltration in deep dermis. e: Vascular impairment with neutrophilic infiltration and nuclear dust in sub cutaneous tissue. f: Mucin deposition in mid dermis. g: Mild and focal thickening of basement membrane. h: Trace and focal deposits of IgG along the dermo-epidermal junction. (a: H\&E, original magnification $\times 40 ; b$ : H\&E, original magnification $\times 100$; c: H\&E, original magnification $\times 100$, d: H\&E, original magnification $\times 400$, e: H\&E, original magnification $\times 400$, f: colloidal iron staining, original magnification $\times 40$, g: PAS staining, original magnification $\times 100$, h: immunofluorescence study, original magnification $\times 100$ ). 


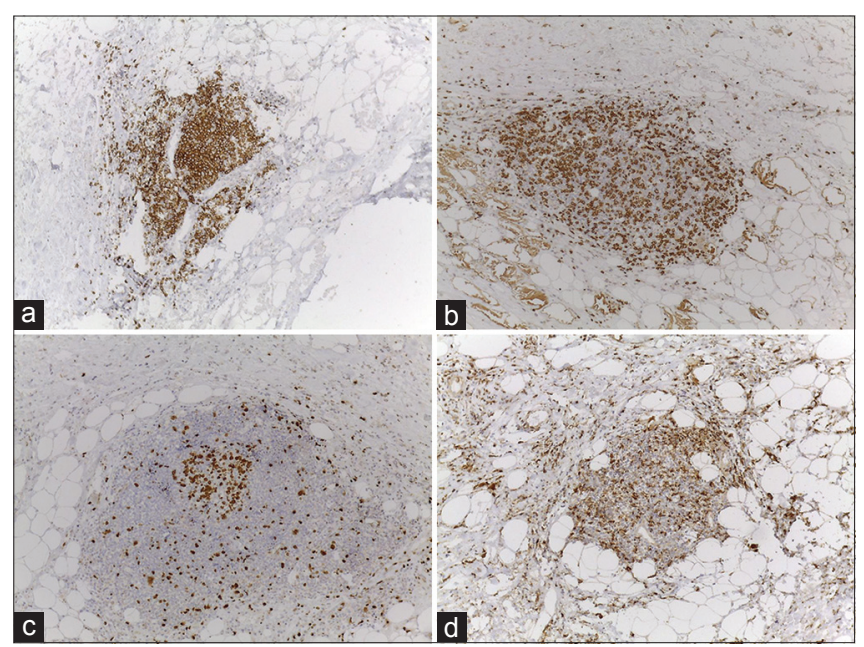

Figure 3: IHC staining results: a: CD 20 is densely positive in $B$ cell component. b: CD3 is positive in concurrent T-cell component. c: In overall Ki67 is positive in $2 \%-4 \%$ of cells, but in about $70 \%$ of cells in germinal center cells. d: CD68 is positive in scattered histiocytes. (3a, b, c, d: original magnification $\times 400$ ).

$182 \mathrm{mg} / 24 \mathrm{hrs}$ (norm up to 150). Urine culture was negative. Imaging studies including chest radiography and abdominopelvic sonography were unremarkable but lesional ultrasonography revealed a hypoechoic region in subcutaneous fat.

According to clinicopathologic correlation and lab data, LEP was proposed. We treated her with oral hydroxychloroquine $400 \mathrm{mg}$ daily for 12 weeks with a marked reduction in pain and induration. She was followed six monthly for any progression to systemic lupus erythematosus (SLE).

\section{DISCUSSION}

LEP is an unusual type of cutaneous lupus erythematosus which could be easily overlooked. Clinically it manifests as indurated subcutaneous painful plaques or nodules usually with normal overlying skin and mainly involves face, trunk, breast and proximal extremities [3,5], although epidermal changes including erythema, hyperpigmentation, poikiloderma, ulceration, discoid lupus erythematosus (DLE)-like features have also been reported $[1,5]$. It mainly affects adults aged between 20 to 60 years and women are almost twice as likely to be affected as men. Seventy percent of LEP cases have been associated with DLE but two to five percent of cases have been accompanied by SLE [6,7]. Most cases of LEP have mild and fluctuant courses but up to $15 \%$ of cases could be able to progress to SLE and $50 \%$ of them have evidences of SLE, so LEP could be a preceding feature of systemic involvement and all patients should be followed up meticulously especially in cases with positive ANA [1,3,6-8]. Arai, et al. reported positive ANA in $95.2 \%$ of LEP cases [8].

Pathophysiology of LEP is not clear but hereditary C4 deficiency and trauma have been incriminated and the role of ultraviolet is questionable because LEP usually involves proximal extremities [3]. In our case, previous trauma could be a triggering factor for antigen presentation. We considered pain in the lesion as a result of neural involvement which was seen in histopathologic evaluation.

Major histopathologic characteristics in LEP are as follows: Lobular lymphocytic panniculitis, lymphoid follicle formation, calcification, hyaline fat necrosis, lipomembranous changes due to vascular impairment and fibrin thrombosis. Other histopathologic characteristics include DLE- like changes in epidermis, mucin deposition ( $80 \%$ of cases), granuloma formation and plasmacytic infiltration. Positive lupus band and basement membrane thickening have been detected in up to $50 \%$ of cases $[3,8,9]$. An important differential diagnosis for LEP is SCTCL. Plasma cell infiltration, lymphoid follicle formation, mucin deposition, DLElike epidermal changes, IHC findings and positive lupus band help to rule out SCTCL $[1,10]$.

We treated her with hydroxychloroquine which is a disease modifier with different mechanisms and few complications [3]. For recalcitrant cases dapsone, thalidomide, cyclosporine and rituximab have been used [1].

\section{CONCLUSION}

An indurated subcutaneous painful plaque could be the presenting feature of LEP. Early diagnosis and appropriate therapy should be considered to prevent disfigurement and progression to systemic involvement.

\section{Abbreviations}

LEP: Lupus erythematosus panniculitis

SCTCL: subcutaneous T cell lymphoma

DLE: discoid lupus erythematosus

SLE: systemic lupus erythematosus

ANA: antinuclear antibody

H\&E: Hematoxylin and eosin

PAS: Periodic Acid Schiff

IHC: Immunohistochemistry 


\section{www.odermatol.com}

\section{REFERENCES}

1. Zhao YK, Wang F, Chen WN, Xu R, Wang Z, Jiang YW, et al. Lupus Panniculitis as an Initial Manifestation of Systemic Lupus Erythematosus: A Case Report. Medicine (Baltimore). 2016;95:e3429.

2. Baltaci M, Fritsch P. Histologic features of cutaneous lupus erythematosus. Autoimmun Rev. 2009;8:467-73.

3. Braunstein I, Werth VP. Update on management of connective tissue panniculitides. Dermatol Ther. 2012;25:173-82.

4. Koley S, Sarkar J, Choudhary SV, Choudhury M, Banerjee G, Bar C, et al. Lupus erythematosus panniculitis: A case report. J Pak Associat Dermatol. 2011;21:118-21.

5. Jacyk WK, Bhana KN. Lupus erythematosus profundus in black South Africans. Int J Dermatol. 2006;45:717-21.

6. Strober BE. Lupus panniculitis (lupus profundus). Dermatol Online J. 2001;7:20.

7. Patel RM, Marfatia YS. Lupus panniculitis as an initial manifestation of systemic lupus erythematosus. Indian J Dermatol. 2010;55:99-101.

8. Arai S, Katsuoka K. Clinical entity of Lupus erythematosus panniculitis/lupus erythematosus profundus. Arai S, Katsuoka K. Autoimmun Rev. 2009;8:449-52.

9. Yamamoto T, Furuhata Y, Tsuboi R. Lipomembranous changes and calcification associated with systemic lupus erythematosus. Clin Exp Dermatol. 2007;32:278-80.

10. Magro CM, Crowson AN, Kovatich AJ, Burns F. Lupus profundus, indeterminate lymphocytic lobular panniculitis and subcutaneous T-cell lymphoma: A spectrum of subcuticular T-cell lymphoid dyscrasia. J Cutan Pathol. 2001;28:235-47.

Copyright by Alizade Narges, et al. This is an open access article distributed under the terms of the Creative Commons Attribution License, which permits unrestricted use, distribution, and reproduction in any medium, provided the original author and source are credited.

Source of Support: Nil, Conflict of Interest: None declared. 